\title{
Prediction of galactic cosmic ray intensity variation for a few (up to 10-12) years ahead on the basis of convection-diffusion and drift model
}

\author{
L. I. Dorman ${ }^{1,2}$ \\ ${ }^{1}$ Israel Cosmic Ray and Space Weather Center and Emilio Segre' Observatory, affiliated to Tel Aviv University, Technion and \\ Israel Space Agency, P. O. Box 2217, Qazrin 12900, Israel \\ ${ }^{2}$ Cosmic Ray Department of IZMIRAN, Russian Academy of Science, Troitsk 142092, Moscow Region, Russia
}

Received: 25 February 2005 - Revised: 24 May 2005 - Accepted: 28 May 2005 - Published: 22 November 2005

Part of Special Issue "1st European Space Weather Week (ESWW)"

\begin{abstract}
We determine the dimension of the Heliosphere (modulation region), radial diffusion coefficient and other parameters of convection-diffusion and drift mechanisms of cosmic ray (CR) long-term variation, depending on particle energy, the level of solar activity (SA) and general solar magnetic field. This important information we obtain on the basis of CR and SA data in the past, taking into account the theory of convection-diffusion and drift global modulation of galactic CR in the Heliosphere. By using these results and the predictions which are regularly published elsewhere of expected SA variation in the near future and prediction of next future SA cycle, we may make a prediction of the expected in the near future long-term cosmic ray intensity variation. We show that by this method we may make a prediction of the expected in the near future (up to 10-12 years, and may be more, in dependence for what period can be made definite prediction of SA) galactic cosmic ray intensity variation in the interplanetary space on different distances from the Sun, in the Earth's magnetosphere, and in the atmosphere at different altitudes and latitudes.
\end{abstract}

Keywords. Interplanetary physics (Galactic cosmic rays; Long-term variation; Modeling and forecasting)

\section{Convection-diffusion modulation}

According to Dorman et al. (2001), the expected value of the natural logarithm of CR intensity global modulation at the Earth's orbit, taking into account the time lag in the Helio-

Correspondence to: L. I. Dorman

(lid@physics.technion.ac.il) sphere relative to the processes on the Sun, will be

$\ln \left(n\left(R, X_{o}, \beta, r_{E}, t\right)_{\exp }\right)=A-B \times F\left(t, X_{o}, \beta,\left.W(t-X)\right|_{X_{E}} ^{X_{o}}\right)$,

where

$$
\begin{aligned}
& \left.\begin{array}{l}
F\left(t, X_{o}, \beta,\left.W(t-X)\right|_{X_{E}} ^{X_{o}}\right. \\
X_{o}
\end{array}\right)= \\
& \quad \int_{X_{E}}\left(W(t-X) / W_{\max }\right)^{\frac{1}{3}+\frac{2}{3}\left(1-W(t-X) / W_{\max }\right)} X^{-\beta} d X, \\
& \begin{array}{l}
X=r / u, X_{E}=1 A U / u, X_{o}=r_{o} / u, \\
\text { and } \\
n\left(R, X_{o}, \beta, r_{E}, t\right)_{\exp }
\end{array}
\end{aligned}
$$

is the expected galactic CR density at the Earth's orbit, in dependence of the values of the parameters $X_{o}$ and $\beta$. Regression coefficients $A\left(R, X_{o}, \beta, t_{1}, t_{2}\right)$ and $B\left(R, X_{o}, \beta, t_{1}, t_{2}\right)$ can be determined by correlation between observed values $\ln \left(n\left(R, r_{E}, t\right)\right)_{\mathrm{obs}}$ and the values of $F\left(t, X_{o}, \beta,\left.W(t-X)\right|_{X_{E}} ^{X_{o}}\right)$, calculated according to Eq. (2), for different values of $X_{o}$ and $\beta$. In Dorman et al. (1997a,b) three values of $\beta=0,0.5,1$, have been considered; it was shown that $\beta=1$ strongly contradicts the CR and SA observation data, and that $\beta=0$ is the most reliable value. Therefore, we will only consider here this value.

\section{Influence of drift effects on the time lag in odd and} even cycles

We suppose that the observed long-term cosmic ray modulation is caused by two processes: the convection-diffusion mechanism (e.g. Dorman, 1959; Parker, 1963; Dorman, 1965), which does not depend on the sign of the solar magnetic field, and the drift mechanism (e.g. Jokipii and Davila, 


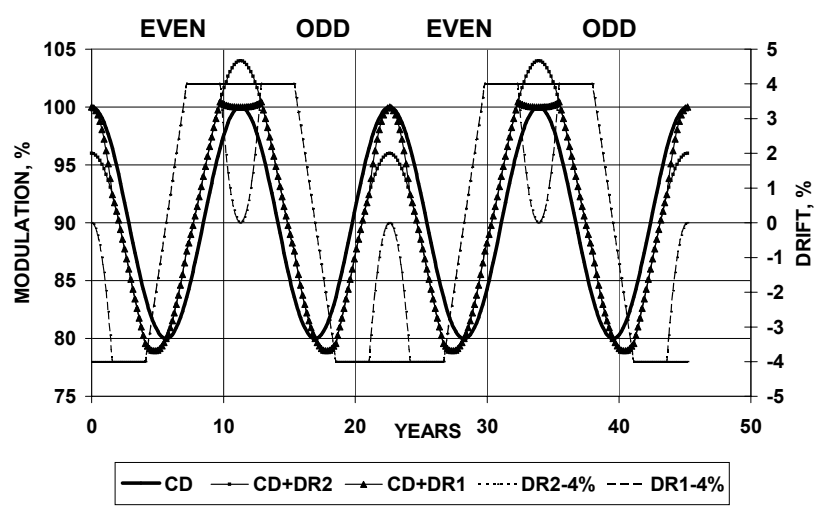

Fig. 1. The first and second approaches of the different influence of drift effects on the observed time lag in odd and even cycles: "CD" is convection-diffusion modulation with a total change of $20 \%$ (as about in Climax NM data), "CD+DR2" and "CD+DR1" are "observed", including convection-diffusion and drift modulations; "DR2-4\%" and "DR1-4\%" are supposed drift effects with amplitude $A_{d r}=4 \%$ (right ordinate).

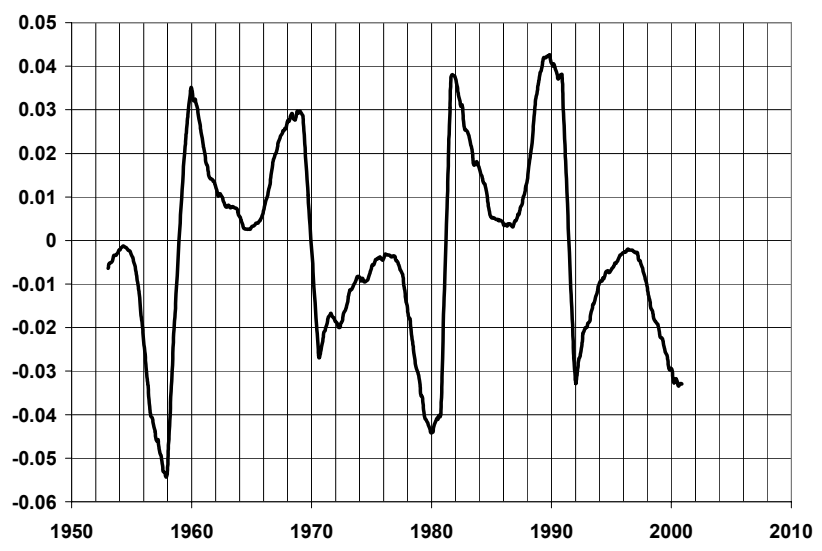

Fig. 2. Drift effect according to the third approach for $A_{d r}=2 \%$ (at $W=75$ ).

1981; Jokipii and Thomas, 1981; Lee and Fisk, 1981; Kota and Jokipii, 1999; Burger and Potgieter, 1999; Ferreira et al., 1999) which gave the opposite effects with a changing sign of solar magnetic field. For the convection-diffusion mechanism we will use the model described in detail in Dorman et al. (2001). We will consider three approaches of drift effects. Two of them are shown schematically in Fig. 1. It can be seen from Fig. 1 that in both approaches drift effects in even cycles lead to a decrease in the time lag and in odd cycles to an increase in the time lag in comparison with that expected in convection-diffusion modulation. The first approach also leads to an increase in the width of the $\mathrm{CR}$ maximum, from an even to odd cycle, but does not change the value of the $\mathrm{CR}$ maximums. The second approach does not change the width of the CR maximums but leads to a relative increase in the CR maximum, from an even to odd cycle, and a decrease from an odd to even cycle (see Fig. 1).

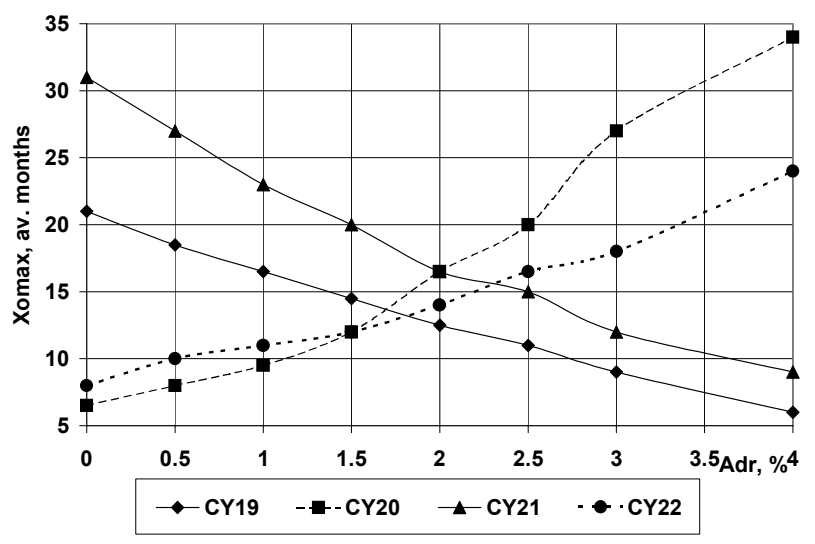

Fig. 3. Dependences $X_{o \max }\left(A_{d r}\right)$ for Climax NM in the frame of the third drift model.

This result contradicts the supposition that the CR intensity out of the modulation region is constant in time. But we cannot exclude this model from consideration and discussion: there can be some additional modulation out of the Heliosphere in periods of solar magnetic field $\mathrm{A}>0$, and no modulation in periods $A<0$ (e.g. only at $A<0$ can be good direct connection of the IMF with the interstellar magnetic field). In the third approach (see Fig. 2) we suppose that the drift effect is proportional to the value of tilt-angle $T$ (see references above on drift effects in galactic CR).

Data were on tilt-angles only for the period between May 1976-September 1993. On the basis of these data it was found that there was a very good connection between $T$ and $W$ : for yearly data $T=0.363 W+13.06^{\circ}$ with correlation coefficient 0.973 , for monthly data $T=0.316 \mathrm{~W}+16.42^{\circ}$ with correlation coefficient 0.882 , and for 11 months of smoothed data $T=0.349 W+13.52^{\circ}$ with correlation coefficient 0.955 . We used 11 months of smoothed data of $W$ and the amplitude $A_{d r}$ of drift effects normalized to $W=75$ (average value of $W$ for the period between January 1953-November 2000). For information on reversal periods we used as follows (according to the Internet): August 1949 \pm 9 months, December $1958 \pm 12$ months, December $1969 \pm 8$ months, March $1981 \pm 5$ months, and June $1991 \pm 7$ months. The drift effect according to the third approach for the period January 1953-November 2000 is shown in Fig. 2 for $A_{d r}=2 \%$ (at $W=75)$. We calculated the correlation coefficients between expected integrals $F$, determined by Eq. (2), with observed "LNCL11M" and "LNHU/HAL11M", as well as for these integrals corrected on drift effects according to the first, second and third models with different amplitudes of the drift effect from $0.15 \%$ up to $4 \%$. As example, in Table 1 are the results of the determination of $X_{o \text { max }}$ and the correlation coefficients for the third model for solar cycles 19, 20, 21, and 22. It can be seen from Table 1 that for odd cycles, increasing of the drift effects leads to a decrease in $X_{o \text { max }}$ but for even cycles situation is inverse: with an increasing in the drift effects $X_{o \text { max }}$ increases. In Figs. 3 and 4 dependences $X_{o \text { max }}\left(A_{d r}\right)$ for Climax NM (sensitive to primary particles with rigidity 


\begin{tabular}{|c|c|c|c|c|c|c|c|c|}
\hline \multicolumn{9}{|c|}{ CLIMAX NEUTRON MONITOR, LN(CL11M) } \\
\hline CYCLE & $\mathbf{0 \%}$ & $0.5 \%$ & $1 \%$ & $1.5 \%$ & $2 \%$ & $2.5 \%$ & $3 \%$ & $4 \%$ \\
\hline 19 & $\mathbf{2 1}, 0.989$ & $\mathbf{1 8 . 5}, 0.987$ & $16.5,0.982$ & $\mathbf{1 4 . 5}, 0.976$ & $\mathbf{1 2 . 5}, 0.968$ & $11,0.958$ & $\mathbf{9 , 0 . 9 4 8}$ & $6,0.924$ \\
\hline 20 & $6.5,0.904$ & $\mathbf{8}, 0.911$ & $\mathbf{9 . 5}, 0.912$ & $\mathbf{1 2}, 0.908$ & $\mathbf{1 6 . 5}, 0.901$ & $\mathbf{2 0}, 0.895$ & $\mathbf{2 7}, 0.893$ & $\mathbf{3 4 , 0 . 8 9 5}$ \\
\hline 21 & $31,0.979$ & $27,0.976$ & $\mathbf{2 3}, 0.972$ & $\mathbf{2 0}, 0.967$ & $\mathbf{1 6 . 5}, 0.963$ & $15,0.946$ & $\mathbf{1 2 , 0 . 9 2 8}$ & $\mathbf{9}, 0.887$ \\
\hline 22 & $\mathbf{8}, 0.955$ & $10,0.960$ & $11,0.964$ & $12,0.965$ & $14,0.964$ & $16.5,0.961$ & $18,0.955$ & $\mathbf{2 4 , 0 . 9 4 1}$ \\
\hline \multicolumn{9}{|c|}{ HUANCAYO/HALEAKALA NEUTRON MONITOR, LN(HU/HAL11M) } \\
\hline CYCLE & $0 \%$ & $0.15 \%$ & $0.25 \%$ & $0.35 \%$ & $0.5 \%$ & \multicolumn{2}{|c|}{\begin{tabular}{l|l}
$0.75 \%$ \\
\end{tabular}} & $1.0 \%$ \\
\hline 19 & $\mathbf{2 0}, 0.971$ & $\mathbf{1 8}, 0.969$ & 16.5,0.966 & $14,0.963$ & $12,0.958$ & \multicolumn{2}{|c|}{$\mathbf{9}, 0.945$} & $6,0.929$ \\
\hline 20 & $\mathbf{1 0 . 5}, 0.881$ & $15,0.883$ & $18,0.880$ & $\mathbf{2 5}, 0.916$ & $\mathbf{3 1}, 0.887$ & \multicolumn{2}{|c|}{ 39,0.899 } & 46,0.912 \\
\hline 21 & $\mathbf{3 4}, 0.929$ & $\mathbf{2 3}, 0.923$ & $18,0.923$ & $\mathbf{1 5}, 0.922$ & $12,0.915$ & \multicolumn{2}{|c|}{$\mathbf{9}, 0.884$} & $7,0.833$ \\
\hline 22 & $\mathbf{9}, 0.978$ & $\mathbf{1 2}, 0.978$ & $11,0.978$ & $\mathbf{1 2}, 0.976$ & $14,0.971$ & \multicolumn{2}{|c|}{$16.5,0.955$} & $22,0.934$ \\
\hline
\end{tabular}

Table 1. Values of $X_{\text {omax }}$ (in av. months, bold) and correlation coefficients for observed data (0\%) and corrected drift effects with different amplitudes, according to the third model.

10-15 GV) and for Huancayo/Haleakala NM (sensitive to 35-40 GV). From Fig. 3 it can be seen that for Climax NM the region of the crossings of the dependences $X_{o \max }\left(A_{d r}\right)$ for the odd cycles with the dependences for the even cycles is very small: $13 \leq X_{o \max } \leq 16.5,1.7 \% \leq A_{d r} \leq 2.3 \%$. For Huancayo/Haleakala NM this region is also very small: $13 \leq X_{o \max } \leq 18,0.23 \% \leq A_{d r} \leq 0.43 \%$ (see Fig. 4). Let us note that for the first and second approaches, the regions of crossings are much bigger than for the third drift approach. Thus, we came to the conclusion that the more reliable is the third drift approach and the amplitude of the drift effects is about $2 \%$ for Climax NM and about $0.25-0.3 \%$ for Huancayo/Haleakala NM.

3 Principles of cosmic ray intensity prediction on the basis of solar activity data and convection-diffusion and drift model

In Fig. 5 a comparison of observed long-term CR variation and corrected on drift effects, according to the third model with amplitude 2\% (at $W=75$ ) for Climax NM (the long-term variation of drift effects for this case was shown in Fig. 2) is shown. Thus, we came to the conclusion that for primary CR with a rigidity of $10-15 \mathrm{GV}$ the relative role of drift effects is about $20 \%$ in periods of high solar activity and negligible near solar minimums. For CR with rigidity $35-40 \mathrm{GV}$, the relative role of drift effects is about 3 times smaller.

From Fig. 5 it can be seen that on the basis of SA data, taking into account convection-diffusion and drift modulations, a very good prediction of CR intensity change can be made, with a correlation coefficient between the observed and predicted intensities of about 0.97 .

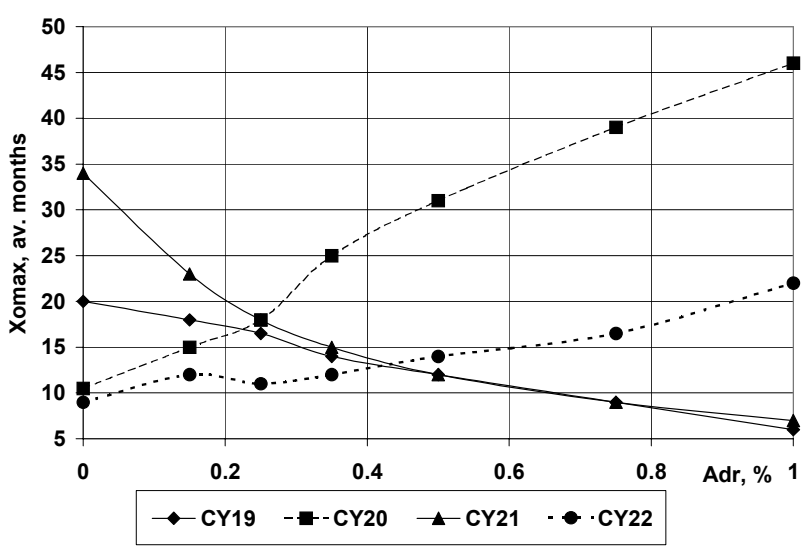

Fig. 4. Dependences $X_{o \max }\left(A_{d r}\right)$ for Huancayo/Haleakala NM.

\section{Prediction of cosmic radiation for few years ahead}

At the onset of the cycle there is no information on the values $X_{o \text { max }}$ and $A_{d r}$ for this cycle, but the type of cycle (odd or even) is known and we can use published predicted values of sunspot numbers for a few years ahead. Thus let us use Eqs. (1) and (2) for convection-diffusion modulation and the third approach for drift effects with values of $X_{o \text { max }}$ and $A_{d r}$ obtained above for cycles 19-22 (data for January 1953December 1995): $X_{o \text { max }} \approx 15$ av. months and $A_{d r} \approx 2 \%$ and $0.25 \%$ for Climax NM (effective rigidity of primary particles 10-15 GV) and Huancayo/Haleakala NM (35-45 GV) accordingly. Predicted and observed CR long-term variations in the onset of cycle 23 are shown in Figs. 6 and 7. Correlation coefficients between predicted and observed cosmic radiation are found 0.988 and 0.986 for Climax NM and Huancayo/Haleakala NM. 


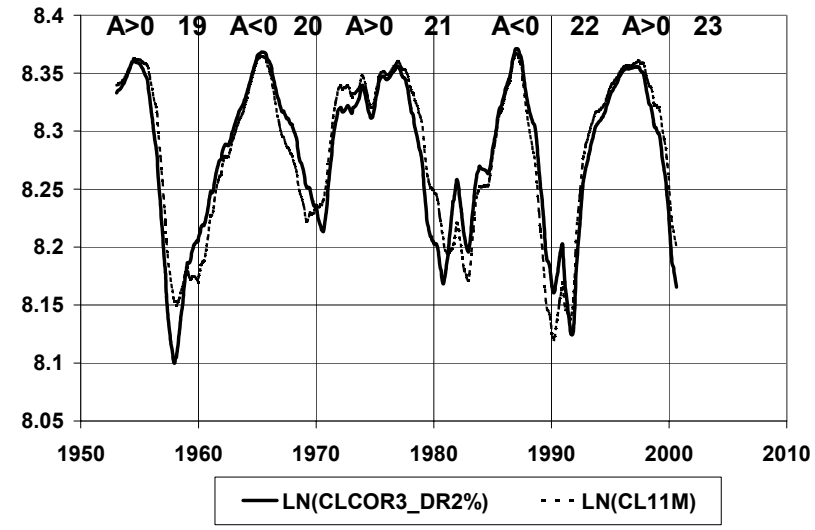

Fig. 5. Climax NM data: comparison of observed LN(CL11M) with expected from convection-diffusion modulation, with corrected drift effects, according to the third approach with $A_{d r}=2 \%$ (at $W=75$ ).

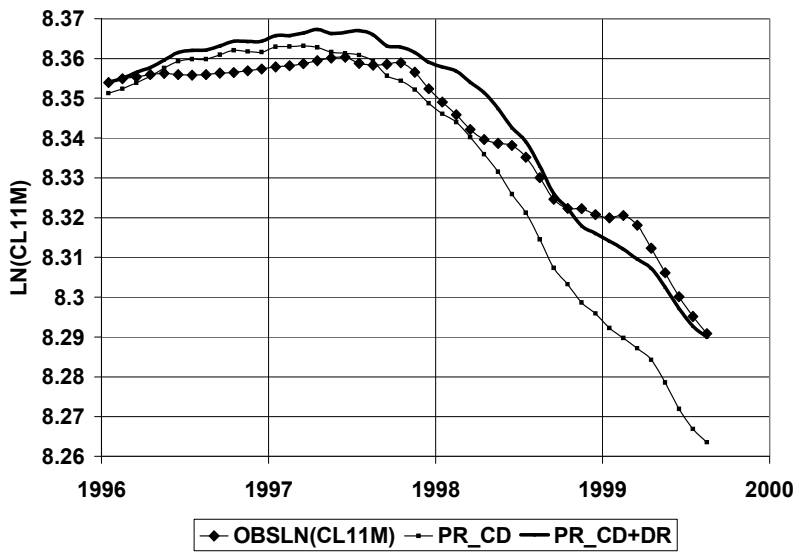

Fig. 6. Comparison of predicted convection-diffusion modulation (PR_CD) and predicted, taking into account drift effects (PR_CD+DR) with observation by Climax NM for the period between January 1996-August 1999

\section{Discussion and conclusions}

Considerating only convection-diffusion long-term modulation leads to a great difference in time lag $X_{o \max }$ for odd and even solar cycles. Taking into account drift effects according to the third model can explain this difference and give the possibility by using data for 19, 20, 21 and 22 solar cycles to estimate the relative role of convection-diffusion and drift mechanisms in the formation of observed long-term $\mathrm{CR}$ modulation. We determined the regression coefficients for the correlation of expected convection-diffusion modulation according to Eqs. (1) and (2), with observed (corrected on drift effects according to the third approach) in the period between January 1953-December 1995. Comparison of the results for $X_{o \text { max }}$ and $A_{d r}$ obtained for odd and even cycles in this period, gave the possibility to determine reliable values for $X_{o \max } \approx 15$ av. months and $A_{d r} \approx 2 \%$ for Climax

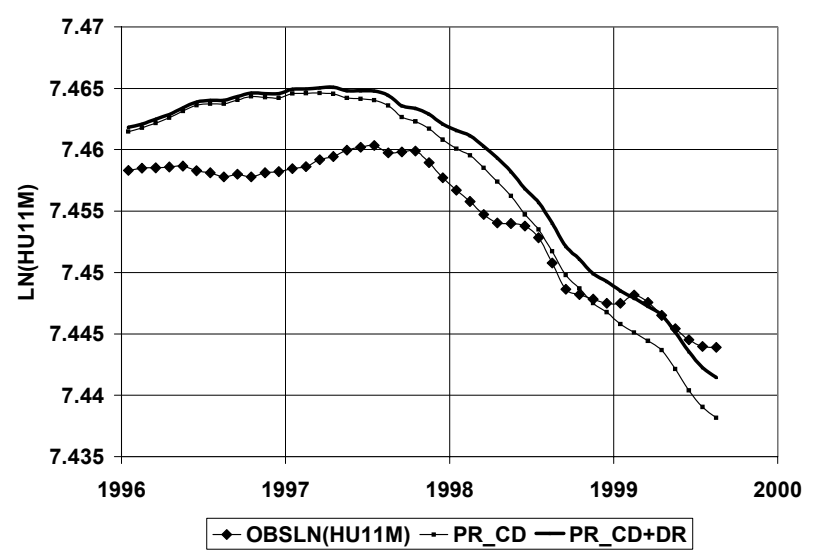

Fig. 7. The same as in Fig. 6, but for Huancayo/Haleakala NM.

NM (effective rigidity of primary particles $10-15 \mathrm{GV}$ ) and $A_{d r} \approx 0.25 \%$ for Huancayo/Haleakala NM (35-45 GV). We came to the conclusion that for Climax NM (effective rigidity of primary particles $10-15 \mathrm{GV}$ ) the convection-diffusion mechanism gives about $80 \%$ of the observed modulation and a drift mechanism of about $20 \%$; for Huancayo/Haleakala NM (effective rigidity of primary particles $35-45 \mathrm{GV}$ ) the relative role of drift effects is about 3 times smaller. By regression coefficients of Eq. (1), estimated for $X_{o \text { max }} \approx 15$ av. months and $A_{d r} \approx 2 \%$ for Climax NM, and $A_{d r} \approx 0.25 \%$ for Huancayo/Haleakala NM we determined the expected convection-diffusion modulation in 1996-1999 only on the basis of monthly sunspot numbers. By adding the expected drift effects, we determined the "predicted" CR intensity. Comparison with observed data gives correlation coefficients 0.988 and 0.986 for Climax NM and Huancayo/Haleakala $\mathrm{NM}$, accordingly. If it is a good prediction of SA for about one solar cycle, correspondingly, then the prediction on the CR intensity variation can be made for about $10-12$ years ahead.

Acknowledgements. Our great thanks to Y. Ne'eman and A. Sternlieb for constant interest and support of the work of Israel Cosmic Ray and Space Weather Center and Emilio Segre' Observatory, and J. Allen, A. V. Belov, E. A. Eroshenko, N. Iucci, J. Lilensten, K. G. McCracken, M. Murat, M. Parisi, L. Pustil'nik, M. A. Shea, D. F. Smart, M. Storini, G. Villoresi, V. G. Yanke, and I. G. Zukerman for collaboration and interesting discussions. The work of neutron monitor on Mt. Hermon is supported by Tel Aviv University, UNIRoma-Tre, and IFSI-CNR Collaboration. This research is partly supported by the European Project COST-724.

Topical Editor T. Pulkkinen thanks two referees for their help in evaluating this paper.

\section{References}

Burger, R. A. and Potgieter, M. S.: The effect of large heliospheric current sheet tilt angles in numerical modulation models: A theoretical assessment, Proc. of 26th Intern. Cosmic Ray Conference, Salt Lake City, 7, 13-16, 1999. 
Dorman, L. I.: To the theory of cosmic ray modulation by solar wind, Proc. of 6-th Intern. Cosmic Ray Conf., Moscow, 4, 328334, 1959.

Dorman, L. I.: Galactic and solar cosmic rays in the interplanetary space, in Proc. of 9-th Intern.Cosmic Ray Conf., London, 1, 292295, 1965.

Dorman L. I., Iucci, N., and Villoresi, G.: Time lag between cosmic rays and solar activity; solar minimum of 1994-1996 and residual modulation, Adv. Space Res., 27, 3, 595-600, 2001.

Dorman L. I., Villoresi, G., I., Dorman, V., Iucci, N., and Parisi, M.: High rigidity CR-SA hysteresis phenomenon and dimension of modulation region in the Heliosphere in dependence of particle rigidity, Proc. 25th Intern. Cosmic Ray Conference, Durban (South Africa), 2, 69-72, 1997a.

Dorman L. I., Villoresi, G., Dorman, I. V., Iucci, N., and Parisi, M.: Low rigidity CR-SA hysteresis phenomenon and average dimension of the modulation region and Heliosphere, Proc. 25th Intern. Cosmic Ray Conference, Durban (South Africa), 2, 7376, 1997b.
Ferreira, S. E. S., Potgieter, M. S., and Burger, R. A.: Comparison of a two and three-dimensional drift model, Proc. 26th Intern. Cosmic Ray Conference, Salt Lake City, 7, 77-80, 1999.

Jokipii, J. R. and Davila, J. M.: Effects of particle drift on the transport of cosmic rays, IV-more realistic diffusion coefficients, Astrophys. J., 248, Part 1, 1156-1161, 1981.

Jokipii, J. R. and Thomas, B.: Effects of drift on the transport of cosmic rays, iv-modulation by a wavy interplanetary current sheet, Astrophys. J., 243, Part 1, 1115-1122, 1981.

Kota, J. and Jokipii, J. R.: Cosmic ray modulation and the structure of the heliospheric magnetic field, in Proc. 26th Intern. Cosmic Ray Conference, Salt Lake City, 7, 9-12, 1999.

Lee, M. A. and Fisk, L. A.: The role of particle drifts in solar modulation, Astrophys. J., 248, Part 1, 836-844, 1981.

Parker, E. N.: Dynamic Processes in the Interplanetary Medium, Intersci. Publ., 1963. 